\title{
Targeting social assistance in protracted crises
}

\author{
Rachel Sabates-Wheeler and Carolina Szyp
}

\author{
February 2022
}

\section{Theme summary}

Targeting social assistance in situations of protracted crises so that it reaches those most in need - rapidly, effectively and without doing further harm - has historically been one of the most complex technical and political challenges for development and humanitarian programmes. Trade-offs beyond technical and economic concerns, raise questions of who how and whether to target at all in contexts of protracted crises due to conflict or recurrent climate shocks. A better understanding of the opportunities and pitfalls of targeting social assistance in these contexts is useful for informing programming across the humanitarian-development spheres of support.

This brief highlights a number of targeting considerations that are unique to, or intensified by, contexts of protracted crises. We showcase the state of the evidence and debate, gaps in the knowledge, and directions for operational research that emerged from the thematic paper on targeting in situations of protracted crises prepared for the inception phase of the BASIC Research programme.

This thematic brief is a shortened version of a BASIC Research Working Paper. To explore this research theme in more detail please refer to:

Sabates-Wheeler, R. and Szyp, C. (2022) Opportunities and Pitfalls of Targeting Social Assistance in Fragile and ConflictAffected Settings (FCAS), BASIC Research Working Paper 12, Brighton: Institute of Development Studies, DOI: 10.19088/BASIC.2022.012

A full list of the references cited in this brief can be found at the BASIC Research Zotero library:

Implemented by

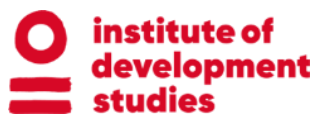

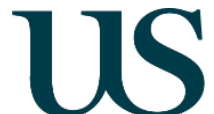

UNIVERSITY OF SUSSEX
UNIVERSITY OF WOLVERHAMPTON

\section{CIDT}

Better Assistance in Crises (BASIC) Research (funded by UKAid) aims to inform policy and programming on how to help poor and vulnerable people cope better with crises and meet their basic needs through more effective social assistance. All costs related to BASIC Research are covered by the UK Foreign, Commonwealth and Development Office. 


\section{State of the evidence and debate}

Debates on targeting are often presented as purely technical, leaving political and social considerations largely unattended. Deciding who is eligible, and how to identify and register these people in protracted crises involves layers of complexity that are less pronounced in stable contexts. Targeting efforts in protracted crisis contexts are characterised by limited information and a dearth of up-to-date data, democratic and institutional deficits, and competing principles and methods of providers, with implications for deciding which targeting methods and data are most appropriate.

In protracted crises, social protection and humanitarian programmes often operate in parallel with potentially overlapping target groups, but also with differences. Duplication, double-dipping, and exclusion could all be minimised through improved humanitarian-development coordination (Ghorpade and Ammar 2021). Harmonisation of targeting in crises can improve gains, but also has economic, political, technical, and security implications and trade-offs.

Building flexibility into programme design and implementation generates opportunities for ensuring the inclusion of those in need in fluid contexts. Flexibility in targeting processes can respond to thirdparty monitoring, grievance, and redress mechanisms, and be enabled through technology and social media. It is also central to ensuring that support is sustained throughout a crisis.

Use of, and access to, technology is particularly relevant in protracted crises, especially where traditional data are lacking. It can help with collecting data, identifying eligible people, early warning, and monitoring. Technologies are used in insecure contexts to scan areas of interest (with satellite imagery), and in contexts of recurring climate shocks through forecasting. Although technology can offer greater transparency, in contexts of fragility it may contribute to a perception that corruption has increased (Transparency International 2016). Risks associated with technologies concern data protection and digital risks and loss of field presence. Equity and effectiveness can be compromised due to the digital gap (Wylde and Venton 2020).

\section{Gaps in the evidence}

Evidence on social assistance targeting processes in protracted crises is limited and patchy - in contrast to the substantial literature on different targeting methods in relatively stable contexts. There is also limited evidence on whether targeting approaches from more stable contexts are appropriate to, or translate to, protracted crisis contexts.

Understanding of the impact of a protracted crisis on targeting effectiveness is poor. Large gaps exist in our understanding of who is in need, to what extent different groups are reached, and how. Little is known about the impacts of crisis or conflict on poverty, who is in need at different times, and how different targeting approaches perform in emergency and humanitarian settings.

Trade-offs between targeting and not targeting social assistance in protracted crises are often unexplored or remain implicit. The literature presents little evidence on the extent to which targeted approaches are more effective, inclusive and considered legitimate compared to approaches like universal targeting and/or lotteries.

Limited research exists on the implications of harmonising targeting processes between humanitarian and state actors. These need to be understood and navigated better in conflict contexts where data privacy and protection risks are heightened. Potential synergies from harmonising also bring risks in contexts where government-led targeting may not be effective. Operating outside government systems of recipient countries can affect targeting effectiveness. 
Evidence is lacking on adapting targeting systems over the course of protracted crises. The fluid dynamics of crisis contexts require frequent re-assessment and adaptation of strategies and actions, yet targeting systems can be inflexible and static. More evidence is needed on designing targeting to flex in line with rapid changes in needs, affected populations and governance at the local level.

\section{Directions for research}

The following research questions emerge from the literature, evidence and debates, as well as from the gaps. These are worth considering for future work in the implementation phase of BASIC Research:

- What are the international, national, and local political landscapes, and the social and power dynamics (in addition to the technicalities) that influence what works in targeting in a specific place at a specific time?

- To what extent is there overlap in targeting by humanitarian assistance and national social protection programmes? To what extent can (or should) humanitarian agencies collaborate or coordinate with governments in protracted crises to achieve any harmonisation within the targeting process? (This question holds particular relevance for provision to refugees and forcibly displaced people.)

- How do protracted crises affect poverty and vulnerability in different settings for different people (such as IDPs and refugees)? How does a context of protracted crisis affect social assistance provision options and performance? How does targeting design and performance vary? How does targeting performance align with perceptions of need and fairness within communities where social assistance is provided?

- What are the barriers to programme uptake and social assistance access for different groups and households affected by protracted crises? What are the options for rapid, simple, and low-cost interventions to reduce administrative, information and security barriers so that those most in need are effectively and safely included in social assistance programmes?

- What are the value-for-money trade-offs of minimising different types of targeting errors in protracted crises? What is the appropriate balance of effort, at different stages of a protracted crisis, between tackling inclusion and exclusion errors? What are economic, efficiency, effectiveness, and equity outcomes of each approach?

\section{Acknowledgements and Disclaimer}

This document was developed by the Better Assistance in Crises (BASIC) Research programme. BASIC is implemented by the Institute of Development Studies (IDS), the University of Sussex and the Centre for International Development and Training, funded by UKAid from the UK government. The views expressed in this document are entirely those of the authors and do not necessarily represent views or policies of the UK governments official policies.

(C) IDS copyright 2022. Copyright in the typographical arrangement and design rests with IDS.

This publication (excluding the logos) may be reproduced free of charge in any format or medium, provided that it is reproduced accurately and not used in a misleading context. The material must be acknowledged as IDS copyright with the title and source of the publication specified.

Published by IDS.

DOI: $10.19088 / B A S I C .2022 .020$ 